\title{
Evolution of the abundance of biomolecules in the interstellar medium at the gas phase
}

\author{
Eduardo M. Penteado and Helio J. Rocha-Pinto \\ Universidade Federal do Rio de Janeiro, Observatório do Valongo, \\ Ladeira Pedro Antônio 43, 20080-090, Rio de Janeiro, Brasil \\ email: monfpent@astro.ufrj.br, helio@astro.ufrj.br
}

\begin{abstract}
Interstellar clouds are the sites where many molecules believed important for the early life are produced. The collapse of such clouds may give birth to stars hosting planetary systems. During the formation of such systems, molecules formed in the molecular cloud, aggregated into grains, can be incorporated in protoplanets, influencing the chemical evolution of the environment, probably affecting the chances for appearance of life on rocky planets located at the stellar habitable zones. Moreover, small bodies, like comets, can carry some of these molecules to inner planets of their systems. Using astrochemical equations, we describe the evolution of the abundance of such molecules at the gas phase from several initial interstellar compositions. These varying initial chemical compositions consider the change of the elemental abundances predicted by a self-consistent model of the chemical evolution of the Galaxy. A system of first order differential equations that describes the abundances of each molecule is solved numerically. This poster describes an innovative attempt to link the astrochemistry equations with the Galactic chemical evolution.
\end{abstract}

Keywords. astrochemistry, astrobiology, molecules, abundances.

\section{Introduction}

Before describing the evolution of molecules at interstellar medium (ISM), we have to know which reactions occur in such environment. Several molecules have been identified in the ISM, leading to laboratory studies of possible reactions that end up with the production of these molecules. Such molecules are continuously created and destroyed, depending on the reactions that they are involved and their rates. The abundance of some molecules can be connected with the abundance of many others. This study is based on the previous work by Herbst \& Klemperer (1973). Here, instead of dealing only with the molecular abundances, we make an attempt to connect the evolution of the abundance of many molecules with the chemical evolution of the Galaxy itself, by using a self-consisted model. This way, we can see how the evolution of these molecules are afected by the Galactic evolution.

\section{Data}

We are working with the OSU 2009 data baset, that contains 6046 reactions and 468 species. To solve the differential equations that describe the evolution of abundances of such molecules, we used the NAHOON $\ddagger$ code. Each one of these molecules has a differential equation that describes the time evolution of its abundance. By varying the initial chemical composition of the interstellar clouds, we can study the behaviour of these

$\dagger$ http://www.physics.ohio-state.edu/ eric/

$\ddagger$ http://www.obs.u-bordeaux1.fr/amor/VWakelam/Valentine\%20Wakelam/Downloads.html 
molecules with the change of some parameters, like metallicity and/or temperature, for example.

\section{Development}

Once we have the database, it is necessary to choose the astrophysical parameters. We use typical values for these parameters, such as $10 \mathrm{~K}$ for the temperature of the molecular cloud, density of $2 \times 10^{5} \mathrm{H} \mathrm{cm}^{-3}$ and ionization rate by cosmic rays of $10^{-17} \mathrm{~s}^{-1}$. We do not consider reactions occuring at the solid-phase, that is, we are working with a database of gas-phase reactions only. The system of differential equations is built by the Nahoon program, using the DLSODE code to solve it. Astrophysical equations are solved to find the elemental abundances of many elements, all reffered to the abundance of $\mathrm{H}$. The solar abundances of these elements are taken from Asplund et al. (2005). The molecular cloud chemical composition is chosen for six metallicities: $[\mathrm{Fe} / \mathrm{H}]=-2.5,-2.0,-1.5,-1.0,-0.5$ and 0.0. This way, we work with six different initial chemical compositions determined by these metallicities. Initially, we consider the cloud is neutral, with all the carbons at CO molecules and all the hidrogens at $\mathrm{H}_{2}$ molecules. The initial compositions were chosen following the predictions of a model for the Galactic chemical evolution (Timmes et al. 1995).

\section{Results}

Important molecules for life are abundantly produced at molecular clouds at the gasphase, being $\mathrm{H}_{2} \mathrm{O}, \mathrm{CO}_{2}, \mathrm{H}_{2} \mathrm{CO}, \mathrm{NH}_{3}, \mathrm{CH}_{4}$ and $\mathrm{HCN}$ the most abundants. Complex molecules, such as cyanopolyynes and many carbon molecules, are likely to be destroyed more efficiently than produced, in the last $90 \%$ of the cloud lifetime, as the metallicity increases. This is so because richer clouds are more oxidant, and the oxygen reacts efficiently with carbons to form other molecules, as $\mathrm{CO}_{2}$, trapping $\mathrm{C}$ molecules that are needed to form more complex molecules. Most of the molecules tend to be destroyed after $10 \%$ of the molecular cloud lifetime - nearly $10^{5}$ years —, regardless of the metallicity, and the rate of destruction is, on average, smaller for lower metalicities. Simple oxygen molecules tend to be more abundant at molecular clouds having solar metallicity, contrary to what can be seen for complex oxygen molecules. Nearly $80 \%$ of the studied molecules reach the peak of their abundances before $10^{5}$ years, suggesting that the molecular cloud collapses before the chemical balance is reached. Comparison with other works (e.g., Shalabiea 2001) show that our model produces similar results for the molecular abundances at the gas phase when we consider a molecular cloud having solar metallicity. However, these estimates are not yet consistent with observations for many molecules studied, showing that only gas-phase reactions underestimate the abundance of several molecules.

\section{References}

Asplund, M., Grevesse, N., \& Sauval, A. 2005, ASP Conference Series, v. 336

Herbst, E. \& Klemperer, W. 1973, ApJ, 185, 505

Shalabiea, O. M. 2001, Astronomy and Astrophysics, 370, 1044

Timmes, F. X., Woosley, S. E., \& Weaver, T. Q. 1995, ApJ Supplement Series, 98, 617 